\title{
Estimation of 3D Geometry of Microtubules Using Multi-angle Total Internal Reflection Fluorescence Microscopy ${ }^{\star}$
}

\author{
Qian Yang ${ }^{1}$, Alexander Karpikov ${ }^{2}$, Derek Toomre ${ }^{3}$, and James Duncan ${ }^{1,2}$ \\ 1 Department of Electrical Engineering \\ 2 Department of Diagnostic Radiology \\ 3 Department of Cell Biology \\ Yale University, New Haven, CT, USA
}

\begin{abstract}
With the ultimate goal to quantify important biological parameters of microtubules, we present a method to estimate the $3 \mathrm{D}$ positions of microtubules from multi-angle TIRF data based on the calibrated decay profiles for each angle. Total Internal Reflection Fluorescence (TIRF) Microscopy images are actually projections of 3D volumes and hence cannot alone produce an accurate localization of structures in the z-dimension, however, they provide greatly improved axial resolution for biological samples. Multiple angle-TIRF microscopy allows controlled variation of the incident angle of the illuminating laser beam, thus generating a set of images of different penetration depths with the potential to estimate the $3 \mathrm{D}$ volume of the sample. Our approach incorporates prior information about intensity and geometric smoothness. We validate our method using computer simulated phantom data and test its robustness to noise. We apply our method to TIRF images of microtubules in $\mathrm{PTK}_{2}$ cells and compare the distribution of the microtubule curvatures with electron microscopy (EM) images.
\end{abstract}

\section{Introduction}

The study of biological processes of microtubules has been greatly aided by total internal reflection fluorescence (TIRF) microscopy. A microtubule is a hollow cylinder, constructed by 13 protofilaments, about $25 \mathrm{~nm}$ in diameter. They have biomedical importance due to their functions as conveyer belts for vesicles inside the cell and to regulate cell migration and division.

The objective-type TIRF microscope has the advantage of commercially accessible and is becoming a powerful tool to study sub-cellular structures. However, we have to calibrate the microscope and measure the axial decay profile. Once properly calibrated, TIRFM provides a high signal to noise ratio by illuminating a limited specimen region immediately adjacent to the substrate. By varying the incident angle, we can obtain TIRF projections with different penetration depths $d$.

\footnotetext{
^ This work is supported in part by a funding from the KECK Foundation.
} 
There is some previous work on estimation of depth/distance information from multi-angle TIRF microscopy images. Truskey et al. collected TIRF images at different penetration depths $d$ for well-spread bovine aortic endothelial (BAEC) stained with a membrane-bound carbocyanine dye, and determined the depth $z$ by using a simplified model of TIRFM optics and fitting the image intensity versus penetration depths 1 . Ölveczky et al. used the inverse Laplace transform to estimate the fluorophore distribution $\mathrm{C}(\mathrm{x}, \mathrm{y}, \mathrm{z})$ and implemented it for a simple geometry and top-hat distribution of fluorohpore(either 0 or 1 ) to determine cell-substrate distances for the whole $x-y$ plane 2. More complex structures like spheres were modeled by Rohrbach to estimate the diameters of isolated granules and the distance between vesicles in the solution and the coverslip 3 . Stock et al. constructed a four-layer model (glass substrate, aqueous extracellular medium, plasma membrane and the cytoplasm) with different refractive indices and used nonlinear regression to calculate the distance between the plasma membrane and the glass substrate 4. Estimations of more complicated 3-D structures have not been attempted to date.

In this study we present a novel method that employs multi-angle TIRF microscopy technique to estimate the geometry of 3D curvilinear structures (e.g. microtubules). Fig 1 is an example of multi-angle TIRF images of microtubules with the penetration depths increasing from left to right. Instrumentation was constructed to calibrate the axial profile of the TIRF microscope thus taking into consideration factors that make the profile deviates from theoretically predicted exponential. A maximum a posteriori (MAP) framework is developed to take TIRF images of different penetration depths as input and estimate $z$ positions along segmented microtubules. The method is validated using a computer generated phantom with known geometrical structure and fluorophore distributions. TIRF imaging process is simulated with the phantom and the resulting multiangle projection images are used as input for 3D estimation. We then apply the method to in vitro microtubules and estimate multiple regions from several data sets. We evaluate the accuracy of the algorithm by imaging microtubules in vitro using different sets of penetration depths and compare the results. The distribution of curvatures along the microtubules are computed and compared with electron microscopy images which are used as ground truth.
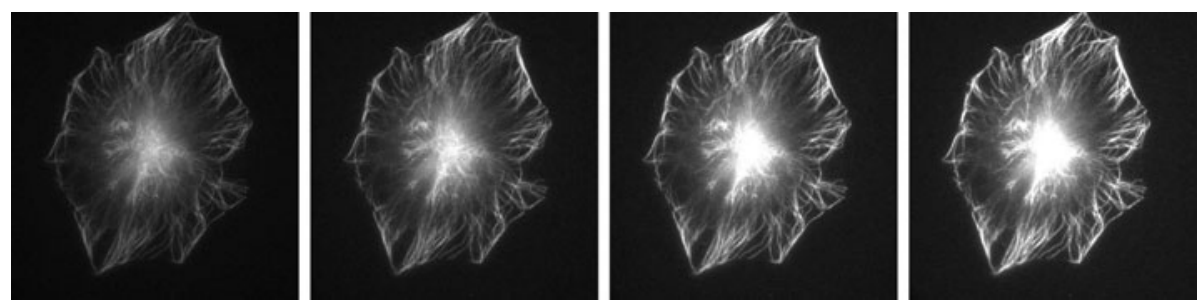

Fig. 1. Multi-angle TIRF images. Left: thinner penetration depth. Right: deeper penetration depth. 


\section{Methods}

\subsection{TIRF Imaging Theory}

An evanescent field is produced when total internal reflection of a laser beam occurs at the interface between a substrate and a sample [5]. Theoretically, the axial profile of the evanescent field behind the interface follows an exponential decay with a single parameter $d$, which refers to the penetration depth:

$$
I(z, d(\theta))=I_{0}(d(\theta)) \cdot e^{-z / d(\theta)}
$$

The penetration depth $d$ is associated with the incident angle:

$$
d(\theta)=\lambda / 4 \pi \cdot\left(n_{i}^{2} \cdot \sin ^{2} \theta-n_{t}^{2}\right)^{-1 / 2}
$$

where $n_{i}$ and $n_{t}$ are the refractive indices of the substrate and the sample, $\theta$ is the incident angle of the laser beam.

The following formula describes how a TIRF image is formed [3]:

$$
I(d(\theta))=\phi I_{0}(d(\theta)) \int_{0}^{\infty}[Q(z) \operatorname{PSF}(z)] C(z) e^{-z / d} \mathrm{~d} z
$$

Here $I$ represents the multi-angle TIRF images we record, $C$ is the actual fluorophore concentration, and $I_{0}(\theta)$ is the intensity directly behind the glass interface. $\phi$ denotes the quantum efficiency of the fluorophores and CCD camera, $Q(z)$ and $P S F(z)$ are the collection efficiency and point spread function.

To calibrate the axial profile and calculate penetration depth, we place a large spherical silicon bead with known diameter on the glass cover slip and place it in the center of the field of laser illumination [6]. For each specific penetration depth, we can combine the projection image with the geometry of the bead to infer the axial decay profile. The calibration result is shown in Fig.2a. Note that the profiles measured are normalized so we also measured $I_{0}(d(\theta))$ to compensate.

\subsection{Challenges in Multi-angle TIRFM}

Assuming a completely constant light source, the photon distribution over a set of bins of equal time is a Poisson probability distribution. Thus, the standard deviation of the number of photon $N$ collected by the CCD camera is $\sqrt{N}$, therefore, the relative uncertainty is given by $\sqrt{N} / N=1 / \sqrt{N}$. This is the major factor leading to falling signal-to-noise ratio along axial direction as we increase the penetration depth to image a thicker part of the sample. Another important factor comes from the background fluorophore in the solution which will also be collected by the camera. Assuming an exponential decay of the axial profile, we calculate the signal-to-noise ratio as a function of depth to be:

$$
S / N \propto\left[\frac{N}{\sqrt{N}}\right] \cdot\left[\frac{1}{\int_{0}^{\infty} e^{-z / d} \mathrm{~d} z}\right]=\sqrt{N} \cdot \frac{1}{d} \propto \frac{e^{-z / 2 d}}{d}
$$




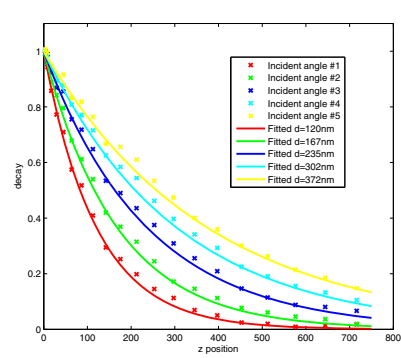

(a)

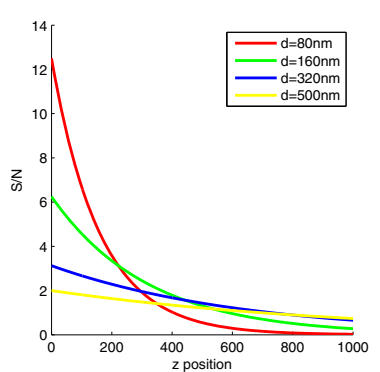

(b)

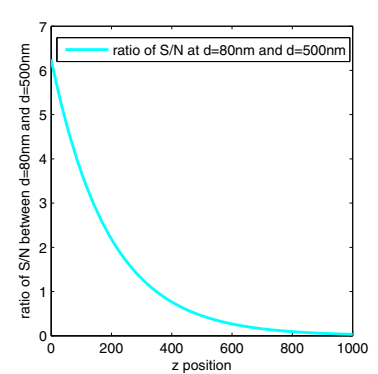

Fig. 2. (a) The decay profiles for different incident angles are calibrated and fitted with exponential decay to find the penetration depths (b) Left: Shot noise/background fluorescence lead to decreasing $\mathrm{S} / \mathrm{N}$, with the decreasing rate inversely proportional to the penetration depth. Right: Ratio of $\mathrm{S} / \mathrm{N}$ between $\mathrm{d}=80 \mathrm{~nm}$ and $\mathrm{d}=500 \mathrm{~nm}$ shows that the latter has a larger value when $z$ is deeper than $350 \mathrm{~nm}$.

where the two terms in the right hand side brackets correspond to the shot noise(photon collecting process) and background fluorescence respectively. This characterizes a major difference between multi-angle TIRF depth estimation from other estimation problems like tomography, where all images are projections of the entire volume and signal-to-noise ratio is the same. With multi-angle TIRFM, there will be a trade-off between the amount of information and the S/N ratio, i.e. as you probe deeper into the sample with increasing penetration depth, $\mathrm{S} / \mathrm{N}$ ratio will drop rapidly. So while it will be a good idea to use images with deep penetration depth as reference images for segmentation purposes, it will be necessary to revisit structures that also exist in low penetration depth images due to better $\mathrm{S} / \mathrm{N}$ ratio. While the low penetration depth images provides good $\mathrm{S} / \mathrm{N}$ ratio for near-the-interface structures, the $\mathrm{S} / \mathrm{N}$ ratio drops considerably faster than deep penetration depth images (Fig,2), so for very deep structures, they won't provide $\mathrm{S} / \mathrm{N}$ ratio as good as the latter since the signal intensity deteriorates dramatically.

\subsection{MAP Framework with Non-informative Prior}

To estimate the $z$ positions for points along the microtubules, we first segment them by manually selecting microtubule tips and finding the shortest path to cells or microtubule organizing centers [7. Since the point spread function will smear out the microtubule to have a width of several pixels, we fit a Gaussian curve along the perpendicular direction to the microtubule to achieve subpixel accuracy of the actual coordinates $[8$. We represent the microtubule centerline represented as $[(x(s), y(s)]$, where $s$ is the curve length. Let $M$ be the number of points sampled along the microtubule, and $N$ be the number of different angles we use to obtain TIRF images. Points where microtubules cross each other are excluded to avoid confusion. A MAP framework is employed to estimate the actual depth information $\left[z_{1}, z_{2}, \ldots, z_{M}\right]$ along the microtubule $\left[\left(x_{1}, y_{1}\right),\left(x_{2}, y_{2}\right), \ldots\left(x_{M}, y_{M}\right)\right]$ 
from a set of TIRF images $\left[I_{1}, I_{2}, \ldots, I_{N}\right]$ corresponding to different penetration depths with axial decay profiles $\left[f_{1}, f_{2}, \ldots, f_{n}\right]$.

Our objective function is the posterior distribution of the depth $z$ :

$$
\begin{aligned}
E & =\log p\left(z \mid I_{1}, \ldots, I_{n} ; f_{1}, \ldots, f_{N}\right) \\
& \propto \log p\left(I_{1}, \ldots, I_{N} \mid z ; f_{1}, \ldots, f_{N}\right) p(z) \\
& =\log \left[\prod_{i=1}^{M}\left(\prod_{j=1}^{N}\left(p\left(I_{j}\left(x_{i}, y_{i}\right) \mid z_{i} ; f_{j}\right)\right)^{w_{j}\left(z_{i}\right) v\left(z_{i}\right)}\right) p\left(z_{i}\right)\right] \\
& =\log \left[\prod_{i=1}^{M}\left(\prod_{j=1}^{N}\left(\frac{f_{j}\left(z_{i}\right)^{I_{j, i}} e^{-f_{j}\left(z_{i}\right)}}{I_{j, i} !}\right)^{w_{j}\left(z_{i}\right) v\left(z_{i}\right)}\right) p_{d}\left(z_{i}\right) p_{g}\left(z_{i}\right)\right]
\end{aligned}
$$

Breaking up the joint density function $p\left(I_{1}, \ldots, I_{N} \mid z ; f_{1}, \ldots, f_{N}\right)$ into products of $p\left(I_{j}\left(z_{i}\right) \mid f_{j}, z_{i}\right)$ is based on an assumption that assumes independence between projection images $I_{j}$ corresponding to different penetration depths, which arises from independence between $f$ 's and $z$ 's. $p\left(I_{j}\left(x_{i}, y_{i}\right) \mid z_{i} ; f_{j}\right)$ is the likelihood function that illustrates the model of the CCD camera photo collection process, which is well approximated by a Poisson distribution. The prior distribution is consist of the depth prior $p_{d}$ and the geometric prior $p_{g}$.

The weight coefficient $w_{j}$ is chosen to be proportional to the $\mathrm{S} / \mathrm{N}$ ratio corresponding to penetration depth $d_{j}$, thus penalizing projections with low reliability and put more weight on

$$
w_{j}\left(z_{i}\right) \propto(S / N)_{j}\left(z_{i}\right)=\frac{N \cdot e^{-z_{i} / 2 d_{j}} / d_{j}}{\sum_{j=1}^{N} e^{-z_{i} / 2 d_{j}} / d_{j}}
$$

The weight coefficient $v$ is chosen to be a increasing function of $z$ to compensate the diminishing likelihood functions as $z$ increases. $v$ is determined heuristically to be a piecewise constant function which exhibits more numerical stability.

Since we have no prior information nor preference on the parameter $z$ we wish to estimate, we use non-informative prior (Jeffreys prior) distribution in our model. It has the property to be invariant under reparameterization $f_{j}$ 's. Jeffreys prior is proportional to the square root of the determinant of the Fisher information:

$$
p_{d}\left(z_{i}\right) \propto \sqrt{\operatorname{det} \mathcal{I}\left(z_{i}\right)}=\sqrt{\sum_{j=1}^{N} \frac{\left(f_{j}^{\prime}\left(z_{i}\right)\right)^{2}}{f_{j}\left(z_{i}\right)}}
$$

It turns out that $p_{d}$ is a decreasing function of $z$, which agrees with our intention to encourage near-substrate positions in many biological circumstances $(e . g$. a cell with a leading edge).

Geometric prior is used to add local smoothness constraint along the microtubules [9], $\alpha$ and $\beta$ control the "elasticity" and "rigidity" of the microtubules:

$$
p_{g}\left(z_{i}\right)=\alpha\left\|\frac{\partial z}{\partial s}\right\|_{\left(x_{i}, y_{i}, z_{i}\right)}^{2}+\beta\left\|\frac{\partial^{2} z}{\partial s^{2}}\right\|_{\left(x_{i}, y_{i}, z_{i}\right)}^{2}
$$

Maximizing the objective function results in our estimation of the depth $z$. 


\section{Results}

\subsection{Computer Simulation Using Phantom Data}

To validate our estimation method, we generate a $128 \times 128 \times 40$ computer simulated phantom with each voxel of size $160 \mathrm{~nm} \times 160 \mathrm{~nm} \times 50 \mathrm{~nm}$, which corresponds to the resolution of the TIRF microscope. We put curvilinear structures in the volume and simulate the TIRF imaging process by projecting the volume into TIRF projection images using different penetrations depths. Background noise is modeled as the ratio of standard deviation of fluorophore concentration in solution and the concentration on the object of interest (assumed to be uniform). Poisson distribution is used to model the photon collecting process of the CCD camera which generates a TIRF image.

We use the set of projection images as input to our method and estimate the depth of the tubular objects. The optimal number of angles used is a tradeoff between single frame estimation accuracy and time resolution of image sequences. Assuming a constant background noise level, we estimate the volume using different number of input angles. As we can see in Fig 3, estimation accuracy using only one input angle is unacceptable. Basically, our algorithm just turn into a trivial case of solving an equation like $I=I_{0} \exp (-z / d)$ if there is just one projection image for a single incident angle. When the number of angle is equal to 2, we begin to see improvements for shallow structures near the interface. Difference between the results from 5 and 10 input angles is not visually detectable.

Robustness is studied by computing depth estimation error versus noise level and depth estimation error versus depth of object of interest. Resulting plots show a linear relation between the estimation error and background noise, which suggests that our algorithm is quite robust to noise, especially when the structures being studied is close to the glass interface. While the target structures get deeper into the evanescent field, we would expect a linear deterioration of estimation accuracy. Fortunately, most of the biological structures we study(plus end of the microtubules) are close to the substrate and generally won't exceed a maximum depth of $1000 \mathrm{~nm}$.

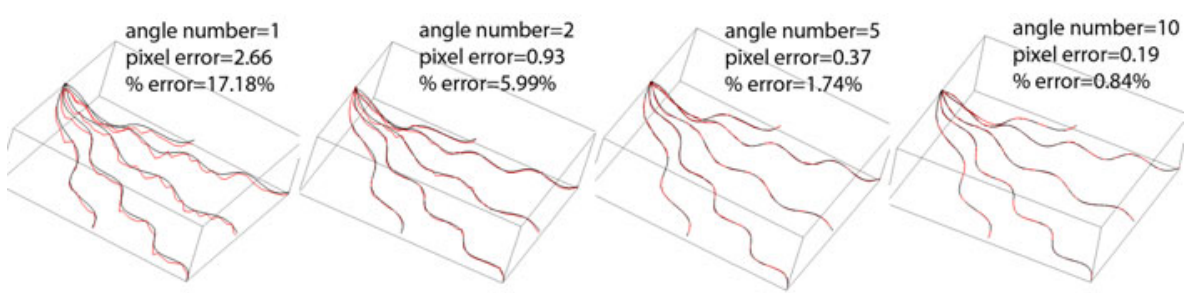

Fig. 3. Estimation error vs number of input angles. Black: Original phantom. Red: Estimated structures. 


\subsection{Estimation Results for in vitro Microtubules}

In our experiments TIRFM images were acquired using an inverted microscope equipped with a high numerical aperture $(\mathrm{NA}=1.495,60 \mathrm{X})$ oil-immersion lens (Olympus) and a back-illuminated electron-multiplying charge-coupled device camera (512 x 512, 16-bit; iXon887; Andor Technologies), and controlled by Andor iQ software (Andor Technology). Excitation was achieved using a 514nm line of argon laser, and exposure times were 0.1-0.2 s. PTK $_{2}$ cells were fixed with $2.5 \%$ paraformaldehyde and $3 \%$ glutaraldehyde in $0.1 \mathrm{M}$ cacodylate buffer ( $\mathrm{pH}$ 7.4) for 2 hours at room temperature, then postfixed for 2 hours at room temperature with $1 \% \mathrm{OsO}_{4}$ in $0.1 \mathrm{M}$ cacodylate buffer ( $\mathrm{pH} 7.4$ ), dehydrated through a series of increasing ethanol concentrations.

Fig 4a and Fig 4d present the estimation results for 5 individual microtubules in a peripheral area of a cell. The $z$ axis in Fig $4 \mathrm{~d}$ is exaggerated for demonstration purpose. In Fig $4 \mathrm{~b}$ we crop out multiple regions of a cell, and estimate them at the same time. Fig 4e shows the 3D view of the estimated volume.

To further validate our method, we pick a single microtubule and repeat the estimation process using different sets of penetration depths. Fig $4 \mathrm{c}$ shows that the estimation results differ by less than the diameter of the microtubules $(25 n m)$, which is considered highly precise. Since the penetration depths and

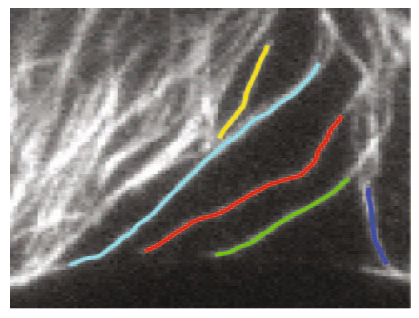

(a)

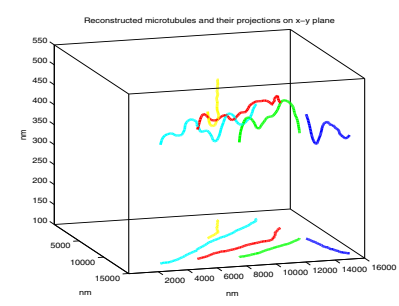

(d)

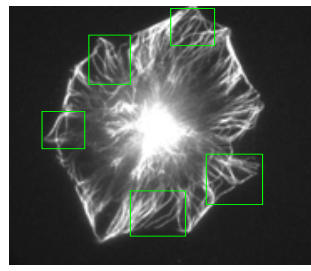

(b)

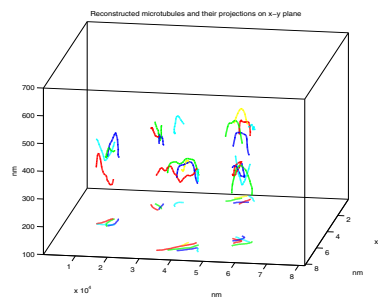

(e)

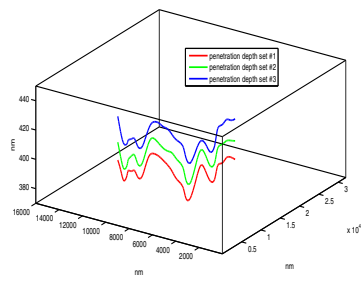

(c)
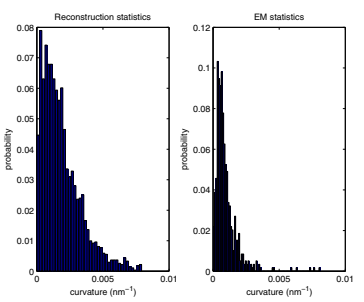

(f)

Fig. 4. (a)\&(d) Estimation results from a single peripheral region. (b)\&(e) Estimation results from multiple peripheral region. A magnified view of the bottom region is shown in (a). (c) Comparison of estimation results using different sets of penetration depth and TIRF images. (f) Comparison of histograms of curvatures from the estimation (left) and EM data (right). 
their corresponding TIRF images are acquired independently, the fact that the estimation results agree with each other further validate our algorithm. Fig 4f compares the distribution of curvatures along the estimated microtubules and in the electron microscopy images we use as a ground truth due to its much larger magnification and the ability to image an axial section. We can see they follow similar distributions, and mostly important, have the same maximum curvature, which is a significant characteristic of microtubules.

\section{Discussion}

The purpose of this study is to develop and validate a method based on MAP framework and optimization techniques to estimate 3-D structures from a set of 2-D projection images generated using multi-angle TIRF microscopy. Our method takes the measured decay curves and corresponding projection images as input, and estimates the axial position of the objects of interest. Computer simulations are used to validate the noise robustness of the algorithm, and also determine the optimal number of input angles we should use. We apply the method to in vitro microtubule images and obtain satisfactory results. The estimation method presented here will be useful for quantifying useful parameters related to microtubules' behavior (movement, growth, etc).

\section{References}

1. Truskey, G.A., Burmeister, J.S., Grapa, E., Reichert, W.M.: Total internal reflection fluorescence microscopy (tirfm) ii. topographic mapping of relative cell/substrate separation distances. Journal of Cell Science 103, 491-499 (1992)

2. Ölveczky, B.P., Periasamy, N., Verkman, A.S.: Mapping fluorophore distributions in three dimensions by quantitative multiple angle-total internal reflection fluorescence microscopy. Biophysics Journal 73, 2836-2847 (1997)

3. Rohrbach, A.: Observing secretory granules with a multiangle evanescent wave microscopy. Biophysical Journal 78, 2641-2654 (2000)

4. Stock, K., Sailer, R., Strauss, W.S.L., Lyttek, M., Steiner, R., Schneckenburger, H.: Variable-angle total internal reflection fluorescence microscopy(va-tirfm): realization and application of a compact illumination device. Journal of Microscopy 211, 19-29 (2003)

5. Axelrod, D., Hellen, E.H., Fulbright, R.M.: Total internal reflection fluorescence. Topics in fluorescence spectroscopy: Principles and applications 3, 289-343 (1992)

6. Mattheyses, A.L., Axelrod, D.: Direct measurement of the evanescent field profile produced by objective-based total internal reflection fluorescence. Journal of Biomedical Optics 11(1) (2006)

7. Cohen, L.D., Kimmel, R.: Global minium for active contour models: A minimum path approach. International Journal of Computer Vision 24(1), 57-78 (1997)

8. Bicek, A.D., Tüzel, E., Demtchouk, A., Uppalapati, M., Hancock, W.O., Kroll, D.M., Odde, D.J.: Anterograde microtubule transport drives microtubule bending in llc-pk1 epithelial cells. Molecular Biology of the Cell 20, 2943-2953 (2009)

9. Kass, M., Witkin, A., Terzopoulos, D.: Snakes: Active contour models. In: International Journal of Computer Vision, 321-331 (1988) 